\title{
LA-SUB--93-198
}

\section{VEPCO Network Model Reconciliation of LANL and MZA Model Data}

Purpose: $\quad$ The LANL DC load flow model of the VEPCO transmission network shows two hundred ten more substations than the AC load flow model produced by MZA Utility Consultants. MZA was requested to determine the source of the difference.

Summary: The AC load flow model used for this study utilizes two standard network algorithms (Decoupled or Newton). The solution time of each is affected by the number of substations. The more substations included, the longer the model will take to solve. In addition, the ability of the algorithms to converge to a solution is affected by line loadings and characteristics. Convergence is inhibited by numerous lightly loaded and electrically short lines.

The MZA model reduces the total substations to 343 by creating equivalent loads and generation. Most of the omitted substations are lightly loaded and rated at $115 \mathrm{kV}$. Appendix 1 lists omitted substations and the substation used as the equivalent.

The MZA model includes sixteen substations not included in the LANL model. These represent new generation including Non-Utility Generator (NUG) sites, additional substations and an intertie (Wake, to CP\&L). There connection to the LANL model is delineated in Appendix 2.

Analysis: 1. All generation was accounted for except VEPC-GS-0110 Cushaw 115 and VEPC-GS-0379 Reusens 138. Cushaw was not included in the model. It is a hydro facility with a rated capacity of $2 \mathrm{MW}$. It was used for approximately $17 \mathrm{GWh}$ in 1991. No reference to generation at Reusens could be found. The LANL DC study rated it at $0 \mathrm{MW}$. It was not modeled.

2. All Interties (VEPC-I- $x x x x$ ) are included as Substations (VEPC-SS$x x x x)$.

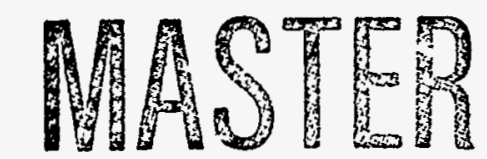

VEPCO Reconciliation $\infty$

hIETMIITINM OF THIS DOCUMENT IS UNLIMITED

December 15, 1992 


\section{DISCLAIMER}

Portions of this document may be illegible in electronic image products. Images are produced from the best available original document. 
3. All substations listed on the LANL DC model were checked against the MZA AC model. For missing substations, the LANL listed transmission lines were reviewed to locate a connection to an MZA listed substation. A list of connections is included as Appendix 1. Finally, a check was made of MZA load against those of LANL. MZA's model shows higher values where several substations were combined. 


\section{VEPCO Network Model Grouped Substations}

Connecting Substation Number Name

VEPC-SS-006 ALLIED 115

VEPC-SS-0051 BURDETTE $02115 \quad 0006$

VEPC-SS-0008 ALTAVISTA 115/NEPC-SS-0009 ALTAVISTA 138 VEPC-SS-0118

VEPC-SS-0150

VEPC-SS-0189

VEPC-SS-0208

VEPC-SS-0262

VEPC-SS-0336

VEPC-SS-0379

DOMINION 115

EVINTON D.P. (SSEC) 115

GRIT (MEC) 115

HICKORY GROVE DP (MEC) 11

LEESVILLE (APCO) 138

OTTER RIVER 115

REUSENS (APCO) 138

VEPC-SS-0023 BATTLEBORO 115

VEPC-SS-0094 CONSOLIDATED STEEL $115 \quad 0023$

VEPC-SS-0292 MAYO DUNBAR (EMEMC) 115

VEPC-SS-0386 ROCKY MTN. (CP\&L) 115

VEPC-SS-0490 WHITAKERS 115

0023-0094

0033

VEPC-SS-0005 ALEXANDER CORNER 115

VEPC-SS-0035 BOYKINS 115

VEPG-SS-0287 NARGARETTSVUKKE 115

VEPC-SS-0476 WATKINS CORNER 115

VEPC-SS-0036 BRADDOCK 230

VEPC-SS-0232 JOHNSON (NVEC) 115

0036

VEPC-SS-0039 BREMO 115/NEPC-SS-0040 BREMO 138/VEPC-SS-0041 BREMO 230

VEPC-SS-0040

VEPC-SS-0046

VEPC-SS-0061

VEPC-SS-0085

VEPC-SS-0227

VEPC-SS-0239

VEPC-SS-0363

BREMO (APCO) 138

BUCKINGHAM (PSE\&G) 230

CARTERVILLE 115

CLIFFORD (APCO) 138

JAMES RIVER IND 115

KIDDS STORE (CVEC) 115

POWHATAN 230

0039

0039-0041

0039-0227

0039-0040

0039

0039

0039-0041

VEPC-SS-0047 BUENA VISA 115

VEPC-GS-0110 CUSHAW 115

VEPC-SS-0016 BALCONY FALLS 115

VEPC-SS-0110 CUSHAW 115

VEPC-SS-0226 JAMES LEE 115
0047-0026-0016

0047-0226

0047-0226-0016

0047 
Attachment 1

\begin{tabular}{cll}
\hline \multicolumn{2}{c}{ VEPC-SS-0056 CANNON BRANCH 115 } & \\
VEPG-SS-0248 & LAKE GASTON 115 & 0056 \\
VEPC-SS-0352 & PLEASANT HILL 115 & $0056-0353$ \\
VEPC-SS-0353 & PLEASANT HILL TAP 115 & 0056 \\
VEPC-SS-0395 & SEABOARD 115 & 0056 \\
VEPC-SS-0498 & WOODLAND 115 & 0056
\end{tabular}

VEPC-SS-0068 CHARLOTTESVILLE 230/NEPC-SS-0069 CHARLOTTESVLLE 115

$\begin{array}{lll}\text { VEPC-SS-0229 } & \text { JARVISBURG 115 } & 0068 \\ \text { VEPC-SS-0108 } & \text { CUNNINGHAM (CVEC) } 115 & 0069\end{array}$

VEPC-SS-0070 CHASE CITY 115

$\begin{array}{lll}\text { VEPC-SS-0018 } & \text { BARNES JCTN (MEC) 115 } & 0070 \\ \text { VEPC-SS-0034 } & \text { BOYDTON (MEC) 115 } & 0070 \\ \text { VEPC-SS-0065 } & \text { CENTRAL 115 } & 0070 \\ \text { VEPC-SS-0170 } & \text { GARY DP.P. (SSEC) 115 } & 0070-0327 \\ \text { VEPC-SS-0235 } & \text { KENBRIDGE 115 } & 0070-0327 \\ \text { VEPC-SS-0324 } & \text { NORTHVIEW (MEC) 115 } & 0070 \\ \text { VEPC-SS-0327 } & \text { NUTBUSH (SSEC) 115 } & 0070 \\ \text { VEPC-SS-0390 } & \text { S. HILL 115 } & 0070-0324 \\ \text { VEPC-SS-0465 } & \text { VICTORIA 115 } & 0070-0327\end{array}$

VEPC-SS-0089 CLUBHOUSE 115

VEPC-SS-0042 BRINK D.P. (MEC) 115

VEPC-SS-0043 BRODNAX 115

VEPC-SS-0045 BRUNSWICK 115

VEPC-SS-0143 EMPORIA 115

VEPC-SS-0144 EMPOIRA (MEC) 115

VEPC-SS-0228 JARRATT 115

VEPC-SS-0259 LAWRENCEVILLE 115

VEPC-SS-0295 METCALF 115

VEPC-SS-0369 PURDY 115

VEPC-SS-0393 SAPONY 115

VEPC-SS-0447 TREGO 115

0089-0295-0143-0447

0089-0259-0045

$0089-0259$

$0089-0265$

0089-0295-0143-0447

0089-0369

0089

0089

0089

0089-0369

0089-0295-0143-0144

VEPC-SS-0102 CREWE 115

VEPC-SS-0165 FT.PICKEIT 115 0102-0166

VEPC-SS-0166 FT PICKETT (SSEC) $115 \quad 0102$

VEPC-SS-0212 HOOPERS D.P. (SSEC) 115

VEPC-SS-0231 JETERSVILLE $115 \quad 0102-0212$

VEPC-SS-0289 MARTINS D.P. (SSEC) 115

VEPC-SS-0113 DEEP CRK 115

VEPC-SS-0172 GILMERTON $115 \quad 0113$

VEPC-SS-0119 DOOMS 115

VEPC-SS-0156 FISHERSVILLE 115

VEPC-SS-0300 MIDWAY (CVEC) $115 \quad 0119$

VEPC-SS-0427 STUARTS DRAFT $115 \quad 0119-0156$

\begin{tabular}{lll}
\hline VEPCO Grouped Substations & $-2-$ & December 15, 1992
\end{tabular} 
Attachment 1

VEPC-SS-0124 DOW 115

VEPC-SS-0290 MARTINS HUNDRED 115

VEPC-SS-0130 DUMFRIES 115

- VEPC-SS-0096 COUNTRY CLUB (NVEC) 1150130

VEPC-SS-0132 DUPONT 115

VEPC-SS-0288 MARLBORO $115 \quad 0132$

VEPC-SS-0133 DUPONT W 115

VEPC-SS-0406 SHERANDO (SVEC) 115

VEPC-SS-0134 EARLEYS 115

VEPC-SS-0234 KELFORD $115 \quad 0134$

VEPC-SS-0137 EDINBURG 115

VEPC-SS-0425 STRASBURG (POTED) $115 \quad 0137-0445$

VEPC-SS-0445 TIMBERVILEE $115 \quad 0137$

VEPC-SS-0139 ELKTON 115

VEPC-SS-0311 N. SHENANDOAH (AEC) 115

VEPC-SS-0142 ELMONT 115

VEPC-SS-0197 HANOVER $115 \quad 0142$

VEPC-SS-0241 KINGS DOMINION (REC) $115 \quad 0142-0197$

VEPC-SS-O149 EVERTTS 115

VEPC-SS-0004

VEPC-SS-0031

ALBEMARLE 115

BETHEL CAROLINA 115

VEPC-SS-0157

FIVE POINTS 115

VEPC-SS-0184 GREENVILE (CP\&L) 230

VEPC-SS-0185 GREENVILLE (CP\&L) 115

VEPC-SS-0263 LEGGETTS 115

VEPC-SS-0264 LEGGETTS X ROADS 115

PARMELE 115

VEPC-SS-0340

PARMELE (EMEMC) 115

0149

0149-0492-0384-0341-0340

$0149-0487$

0149-0487-0263-0185

0149-0487-0263

0149-0487

0149-0487-0263

0149-0492-0384-0341

0149-0492-0384

VEPC-SS-0384

ROBERSONVILLE 115

$0149-0492$

0149-0487-0203-0264

0149

0149

VEPC-SS-0153 FARMVILLE 115

VEPC-SS-0109 CURDSVILLED.P.(CVEC) 115

VEPC-SS-0303 MORAN D.P. (SSEC) 115

VEPC-SS-0163 FREDERICKSBURG 115

$\begin{array}{lll}\text { VEPC-SS-0309 } & \text { N. DOSWELL (REC) 115 } & 0163-0412-0499-0419 \\ \text { VEPC-SS-0412 } & \text { SLABTOWN D.P. (REC) 115 } & 0163 \\ \text { VEPC-SS-0419 } & \text { ST. JOHNS 115 } & 0163-0412-0499 \\ \text { VEPC-SS-0420 } & \text { STAFFORD 115 } & 0163\end{array}$


VEPC-SS-0499 WOODPECKER DP (REC) 115

VEPC-SS-0177 GORDONSVILLE 115

VEPC-SS-0364 PRATTA (APCO) 115

VEPC-SS-0182 GREAT BRIDGE 115

VEPC-SS-0090 COMMN WLTH NAT GAS 115

VEPC-SS-0193 HALIFAX 115

VEPC-SS-0060

VEPC-SS-0083

VEPC-SS-0087

VEPC-SS-0105

VEPC-SS-0127

VEPC-SS-0196

VEPC-SS-0240

VEPC-SS-0334

VEPC-SS-0374

VEPC-SS-0388

VEPC-SS-0411

. VEPC-SS-0480

\section{CARTER FABRICS 115}

CLARSVILLE 115

CLOVER 115

CRYSTAL HILL (MEC) 115

DRYBURG 115

HALIFAX WORSTED 115

KINDERTON 115

OMEGA D.P. (MEC) 115

REEDY CRK 115

S. BOSTON 115

SINAI 115

WLCO 115

VEPC-SS-0194 HALIFAX 230

VEPC-SS-0346 PERSON (CP\&L) 230

VEPC-SS-0198 HARMONY VILLAGE 115

VEPC-SS-0491 WHITE STONE 115

VEPC-SS-0201 HARRISONBURG 115

N. RIVER D.P. (SVEC)

VEPC-SS-0202 HARROWGATE 115

VEPC-SS-0471 WALTHALL 115

VEPC-SS-0203 HARVELL 115

VEPC-SS-0160 FORT LEE 115

VEPC-SS-0211 HOLLYMEAD 230

VEPC-SS-0063 CASHS CORNER (CVEC) 230

VEPC-SS-0507 CASHS CORNER (CVEC) 115

VEPC-SS-0214 HONERTOWN 115

VEPC-SS-0146 ENFIELD (HEMC) 115

VEPC-SS-0195 HALIFAX (REMC) 115

VEPC-SS-0218 IBM 115

VEPC-SS-0003

VEPC-SS-0055

VEPC-SS-0175

VEPC-SS-0285
AIRPORT 115

CANNON BRANCH 115

GOWIND (NVEC) 115

MANASSAS 115
0163-0412

0177

0182

0193-0374

0193-0374-0334

0193

0193

0193-0087

0193

0193-0374-0333-0383

0193-0374

0193

0193-0196-0411

0193-0196

0193-0196-0411

0194

0198

0201

0202

0203

0211

0211-0063

0214-0195

0214

0218

0218-0003

0218

0218-0003-0055 
Attachment 1

VEPC-SS-0481 WELLINGTON (NVEC) 115

VEPC-SS-0500 WOODS 115

VEPC-SS-O219 ICI 115

VEPC-SS-0147 ENON 115
0218-0175

0218-0003-0055-0285

0219

VEPC-SS-0230 JEFFERSON ST. 230NEPC-SS-0231 JEFFERSON ST 115

VEPC-SS-0268 LINDENDALE (NVEC) 115

VEPC-SS-0302 MINNIEVILLE (NVEC) 115

VEPC-SS-0414 SMOKETOWN (NVEC) 115

0230-0414-0302

0230-0414

0230

VEPC-SS-0237 JERR DAM 115

VEPC-SS-0084 CLARKSVILL (MEC) 115

VEPC-SS-0206 HENDERSON (CP\&L) 115

VEPC-SS-0224 ISLAND CRK (GOV. OWN) 115

VEPC-SS-0236 KERR D.P. (MEC) 115

VEPC-SS-0269 LITTLETON (CP\&L) 115

0237-0236-0224

0237

0237-0236

0237

0237

VEPC-SS-0254 LANCASTER 115

VEPC-SS-0169 GARNER D.P. (NNEC) 115

0254

VEPC-SS-0258 LANEXA 115

VEPC-SS-0180 GRAFTON 115

VEPC-SS-0317 NEWPORT RES. $115 \quad 0258$

VEPC-SS-0260 LEBANON 115

VEPC-SS-0159 FORT EUSTIS $115 \quad 0260-0261$

VEPC-SS-0261 LEE HALL 115

0260

VEPC-SS-0506 YORKTOWN NAV. WPNS 115

0260

VEPC-SS-0266 LEXINTON (APCO) 500

VEPC-SS-0508 CLOVERDALE (APCO) $500 \quad 0266$

VEPC-SS-0272 LOCKS 230

VEPC-SS-0460 UNIONVILLE (REC) $115 \quad 0272$

VEPC-SS-0275 LONG CREEK 115

VEPC-SS-0024 BAYSIDE 115

0275

VEPC-SS-0276 LOUDOUN 115

VEPC-SS-0013 ARCOLA (NVEC) $115 \quad 0276$

VEPC-SS-0064 CARHARPIN (NVEC) 115

VEPC-SS-0106 CUB RUN (NVEC) $115 \quad 0276$

VEPC-SS-0167 GAINSVILL $230 \quad 0276-0277$

VEPC-SS-0286 MANCHESTER 115

VEPC-SS-0291 MAURY ST.IND, $115 \quad 0286$

VEPC-SS-0358 PORTER ST 115 0286

VEPC-SS-0298 MIDLOTHIAN 230

\begin{tabular}{lll}
\hline VEPCO Grouped Substations & $-5-$ & December 15,1992
\end{tabular} 
Attachment 1

\begin{tabular}{|c|c|c|c|}
\hline $\begin{array}{l}\text { VEPC-SS-0158 } \\
\text { VEPC-SS-0299 } \\
\text { VEPC-SS-0349 }\end{array}$ & $\begin{array}{l}\text { FLAT ROCK } 115 \\
\text { MIDLOTHIAN } 115 \\
\text { PLANTATION } 115\end{array}$ & $\begin{array}{l}0298-0299 \\
0298 \\
0298-0299\end{array}$ & \\
\hline \multicolumn{4}{|c|}{ VEPC-SS-0315 NEWPORT NEWS 230} \\
\hline VEPC-SS-0028 & BENNS CHURCH 230 & 0315 & \\
\hline \multicolumn{4}{|c|}{ VEPC-SS-0328 OAK GROVE 115} \\
\hline VEPC-SS-0392 & SANDERS D.P. (NNEC) 115 & 0328 & \\
\hline \multicolumn{4}{|c|}{ VEPC-SS-0330 OAKWOOD 115} \\
\hline VEPC-SS-0372 & RED HILL (CVEC) 115 & 0330 & \\
\hline \multicolumn{4}{|c|}{ VEPC-SS-0331 OCCOQUAN 230} \\
\hline VEPC-SS-0332 & OCCOQUAN 115 & 0331 & \\
\hline \multicolumn{4}{|c|}{ VEPC-SS-0335 ORANGE 115} \\
\hline VEPC-SS-0122 & DOUBLEDAY (CVEC) 115 & 0335 & \\
\hline \multicolumn{4}{|c|}{ VEPC-SS-0337 OX (PJM) 500} \\
\hline VEPC-SS-0050 & BURCHES HILL (PELEC) 500 & 0337 & \\
\hline \multicolumn{4}{|c|}{ VEPC-SS-0339 PAMPLIN 115} \\
\hline VEPC-SS-0373 & RED HOUSE (SSEC) 115 & 0339 & \\
\hline VEPC-SS-0389 & S. CRK 115 & 0339 & \\
\hline VEPC-SS-0454 & TWITTYS CRK 115 & 0339 & \\
\hline \multicolumn{4}{|c|}{ VEPC-SS-0344 PENINSULA 115} \\
\hline VEPC-SS-0313 & NASA (POR) 15 & 0344 & \\
\hline VEPC-SS-0459 & UNION CARBIDE 115 & 0344 & \\
\hline \multicolumn{4}{|c|}{ VEPC-SS-0350 PLAZA 115} \\
\hline VEPC-SS-0026 & BELLWOOD 115 & $0350-0314$ & \\
\hline VEPC-SS-0044 & BROWN-BOVERI 115 & $0350-0314-0026$ & \\
\hline VEPC-SS-0245 & KINGSLAND 115 & $0350-0314-0044$ & \\
\hline VEPC-SS-0314 & NAT CYL. GAS 115 & 0350 & \\
\hline VEPC-SS-0380 & REYMET 115 & $0350-0314-0044-0245$ & \\
\hline \multicolumn{4}{|c|}{ VEPC-SS-0355 PLYMOUTH 115} \\
\hline VEPC-SS-0101 & CRESWELL 115 & $0355-0283$ & \\
\hline VEPC-SS-0283 & MACKEYS 115 & 0355 & \\
\hline \multicolumn{4}{|c|}{ VEPC-SS-0357 POE 115} \\
\hline VEPC-SS-0117 & DISPUTANIA 115 & 0357 & \\
\hline VEPC-SS-0225 & IVOR 115 & 0357 & \\
\hline VEPC-SS-0477 & WAVERLY 115 & $0357-0117$ & \\
\hline \multicolumn{4}{|c|}{ VEPC-SS-0377 REMINGTON 115} \\
\hline VEPC-SS-0221 & IND HILL (NVEC) 115 & $0377-0418$ & \\
\hline VEPC-SS-0418 & SOWEGO (NVEC) 115 & 0377 & \\
\hline
\end{tabular}


VEPC-SS-0385 ROCKBRIDGE 115

VEPC-SS-0054 BUSTLEBURG DP (BARC) 115

VEPC-SS-0394 SCOTLAND NECK 115

VEPC-SS-0391 SAMS HEAD (HEMC) 115

VEPC-SS-0402 SHAWBORO 115

VEPC-SS-0017 BARCO 115 0402

VEPC-SS-0368 PT. HARBOR $115 \quad 0402$

VEPC-SS-0404 SHELLBANK 115

VEPC-SS-0030 BETHEL (NVEC) 115

VEPC-SS-0408 SHOCKOE 115

VEPC-SS-0479 WECO $115 \quad 0408$

VEPC-SS-0409 SHORT PUMP 115

VEPC-SS-0188 GREENWWOOD D.P. (REC) $115 \quad 0409$

VEPC-SS-0422 STAUNTON 115

VEPC-SS-0037 BRAND D.P. (SVEC) 115

VEPC-SS-0099 CRAIGSVILLE $115 \quad 0422-0448$

VEPC-SS-0178 GOSHEN 115 0422-0448-0099

VEPC-SS-0448 TRIMBLES MILLDP(SVEC) $115 \quad 0422$

VEPC-SS-0464 VERONA $115 \quad 0422$

VEPC-SS-0486 WEYERS CAVE $115 \quad 0422-0464$

VEPC-SS-0426 STRATFORD HILLS 115

VEPC-SS-0066 CENTRALIA 115

VEPC-SS-0430 SUFFOLK 115

VEPC-SS-0210 HOLLAND 115

VEPC-SS-0242 KINGS FORK $115 \quad 0430-0329$

VEPC-SS-0308 MYRTLE $115 \quad 0430-0329$

VEPC-SS-0329 OAK RIDGE $115 \quad 0430$

VEPC-SS-0468 WAKEFIELD 115 0430-0329-0308

VEPC-SS-0431 SULLY 115

VEPC-SS-0082 CLARK $115 \quad 0431-0128$

VEPC-SS-0128 DULLES 115

VEPC-SS-0432 SURRY 230

VEPC-SS-0181 GRAVEL NECK 230

VEPC-SS-0437 TARBORO 115

VEPC-SS-0011 ANACONDA $115 \quad 0437-0435$

VEPC-SS-0093 CONETONE (EMEMC) $115 \quad$ 0437-0438

VEPC-SS-0435 TAR RIVER $115 \quad 0437$

VEPC-SS-0438 TARBORO (EMEMC) $115 \quad 0437$ 
VEPC-SS-0450 TROWBRIDGE 115

VEPC-SS-0485 WEYERHAUSER 1150450

VEPC-SS-0451 TUNIS 115

VEPC-SS-0002 AHOSKIE 115

0451

VEPC-SS-0453 TWELFTH ST. 115

VEPC-SS-0348 PIPELINE 115

VEPC-SS-0458 UNION CAMP 115

VEPC-SS-0162 FRANKLIN 115

VEPC-SS-0469 WALLER 230

VEPC-SS-0446 TOANO $115 \quad 0469-0470$

VEPC-SS-0470 WALLER $115 \quad 0469$

VEPC-SS-0473 WARRENTON 115

VEPC-SS-0038 BRANDY (REC) 115

VEPC-SS-0482 WEST POINT 115

VEPC-SS-0400 SHACKLEFORD 115

VEPC-SS-0494 WINFALL 115

VEPC-SS-0075 CHOWAN 115

VEPC-SS-0136 EDENTON 115

0494

0494-0075 


\section{VEPCO Network Model \\ MZA Unlisted Substations}

MZA Substation

LANL Substation Number and Name

7645 BELLEMEADE 230

VEPC-SS-0021 BASIN 230

7961 COLINGTON 115

VEPC-SS-0246 KITTY HAWK 115 and VEPC-SS-0312 NAGS HEAD 115

7705 DARBYTOWN 230

VEPC-SS-0073 CHICKAHOMINY 230 and VEPC-SS-0321 NORTHEAST 230

7495 EDGECOMBE NUG 230

VEPC-SS-0387 ROCKY MTN (CP\&L) 230 and VEPC-SS-0436 TARBORO 230

4798 ELIZABETH RIVER 230

VEPC-SS-0497 WOODBRIDGE 230

7127 GLEN CARLYNT 230

VEPC-SS-0151 FALLS CHURCH 230

7309 HALL BRANCH 115

VEPC-SS-0008 ALTAVISTA 115 and VEPC-SS-0193 HALLIFAX 115

7706 LIGHTFOOT 230

VEPC-SS-0244 KINGS MILL 230

7141 PENTAGON 230

VEPC-SS-0173 GLEBE 230

7436 ROSEMARY NUG 230

VEPC-SS-0215 HORNERTOWN 230

7455 SOUTHHAMPTON 115

VEPC-SS-0035 BOYKINS 115 and VEPC-SS-0458 UNION CAMP 115

4755 SOUTH ANNA 230

VEPC-SS-0176 GORDONSVILLE 230 and VEPC-SS-0279 LOUISA 230

7111 STERLING PKT 230

VEPC-SS-0354 PLEASANT VEW (PELEC) 230

7204 STUARTS HILL 115

VEPC-SS-0119 DOOMS 115 and VEPC-SS-0133 DUPONT 115

7187 VA. HIILLS 230

MZA Unlisted Substations

$-1-$

December 15, 1992 
Attachment 2

VEPC-SS-0205 HAYFIELD 230 and VEPC-SS-0230JEFFERSON ST. 230

9 WAKE 500

VEPC-SS-0059 CARSON (CP\&L) 500 
May 6, 1993

University of California

Los Alamos National Laboratory

Attn: J.F. Roach

Reference: 9-LY2-W6097-1

Mail Stop 299

P.O. Box 1663

Los Alamos, NM 87545

Dear Mr. Roach:

Subject: Transmittal of Duke Power Load Flow Model

Enclosed is a copy of the Duke Power Company AC load flow model and related database. The model was constructed from a Duke Power Company System Planning Department Operating Study and updated with information received from neighboring utilities and utility contacts.

We were unable to acquire transmission line information for the $115 \mathrm{KV}$ segments. The $115 \mathrm{kV}$ lines belong to interconnected utilities and the Duke model represents them as generation or load at the relevant Duke bus. A listing is enclosed as Appendix 1.

Several of the substations listed in the Los Alamos DC study have been combined. In general this was done to simplify configurations for substations that were taps or very close to a larger substation. A list of missing substations and the modeled substation is included as Appendix 2. Appendix 3 lists substations in the MZA database not included in the LANL database.

A copy of this letter and all attachments except the model, database and hand drawn diagram have been forwarded to Mr. Michael Pierotti.

If you have any questions, please contact me at (213) 299-7561.

Thank you;

Michele Wynne

Principal 
Appendix 1

Modelling of $115 \mathrm{kV}$ Substations

0010 ASHVILLE CP\&L

0031 BUSH RIVER

0033 CAMDEN CP\&L

0036 CANTON CP\&L

0046 CLARK HILL

0054 DAN RIVER

0058 DAVIDSON RIVER DUM CP\&L

0091 HORSESHOE

0168 PISGAH

0171 RIDEWAY

0182 SALUDA

0204 WATEREE

0208 WESTMINSTER GAP
3152 HORSESHOE

3408 BUSH RIVER

3450 WATEREE

3130 PISGAH

3175 CLARK HILL

3151 DAN RIVER

3150 DAVIDSON RIVER

3152 HORSESHOE

3130 PISGAH

3350 DAN RIVER

3408 BUSH RIVER

3450 WATEREE

3150 WESTMINSTER 
Appendix 2

LANL Substations not in MZA Model

\begin{tabular}{|c|c|c|}
\hline 0001 ABBOTTS CRK & 3368 & LEXINGTON \\
\hline 0019 BLACKSBURG & 3404 & SHELBY \\
\hline O020 BLUE RIDGE COOP & 2206 & BLUE RIDGE 230 \\
\hline 0021 BLUE RIDGE COOP & 2206 & BLUE RIDGE 230 \\
\hline O028 BURLINGTON & 3371 & MEBANE \\
\hline O034 CAMPO BELLO & 3153 & HENDERSONVILLE \\
\hline 0035 CANE CRK & 3161 & E. GREENVILLE \\
\hline 0038 CEDAR CRK HYDRO & 3451 & GREAT FALLS \\
\hline 0042 CHESNEE & 3123 & PEACH VALLEY \\
\hline 0049 CLOVER & 3405 & PEACOCK \\
\hline 0057 P.H.GLATFELTER CORP & 3450 & DAN RIVER \\
\hline 0059 DEARBORN HYDRO & 3451 & GREAT FALLS \\
\hline 0060 DIXIE & 34.61 & HILLTOP \\
\hline 0062 DURHAM PARK & 3303 & ENO \\
\hline 0065 EASLEY & 2206 & BLUE RIDGE 230 \\
\hline 0070 FISHING CREEK HYDRO & 3451 & GREAT FALLS \\
\hline 0073 GRAND CREEK TAP & 3315 & BUCK \\
\hline 0075 GREENLAWN & 2206 & BLUE RIDGE \\
\hline 0079 GREER & 3161 & E. GREENVILLE \\
\hline 0085 HIGHTOWER & 3161 & E. GREENVILLE \\
\hline 0092 IDOLS TAP BENT & 3257 & POPLAR SWITCH \\
\hline 0099 LAUREN ELECTRIC COOP & 3161 & E. GREENVILLE \\
\hline 0100 LAURENS & 3109 & SHADY GROVE \\
\hline 0102 LEE STEAM PLT. & 3109 & SHADY GROVE \\
\hline 0109 MADISON & 3350 & DAN RIVER \\
\hline 0110 MARION & 3250 & BRIDGEWATER \\
\hline 0118 MIDWAY & 3400 & NEWPORT \\
\hline 0122 MOCKSVILLE & 3257 & POPLAR SWITCH \\
\hline 0128 MOTLEY & 3350 & DAN RIVER \\
\hline 0129 MT ISLAND HYDRO & 3464 & RIVERBEND \\
\hline 0136 NEWBERRY & 3400 & NEWPORT \\
\hline 0140 NEWTON & 3258 & HICKORY \\
\hline 0158 PICKENS & 2206 & BLUE RIDGE 230 \\
\hline 0159 PINK HARRILL & 3125 & CLIFFSIDE \\
\hline 0160 PINNACLE & 3260 & BANNERTOWN \\
\hline 0163 AMERICAN THREAD & 3151 & DAVIDSON RIVER \\
\hline 0178 RUTLEDGE & 3260 & BANNERTOWN \\
\hline 0183 SANDY SPRINGS & 3408 & BUSH \\
\hline 0184 SENECA & 3104 & CENTRAL \\
\hline 0193 & 3257 & $\mathbf{P}$ \\
\hline
\end{tabular}


0196 TAYLORSVILLE 0201 BALDESE 0209 WILDCAT 0210 WILKES
3261 N. WILKESBORO

3251 BREEDEN

3464 RIVERBEND

3261 N. WILKSBORO 
Appendix 3

$$
\text { MZA Substations on on LANL Listing }
$$

401 RICHMOND 500

2103 KEOWEE

2106 SEPA HARTWELL 2107 SOUTHERN BIO

2302 BRASSFIELD

2316 BOBWHITE

2350 ROXBORO

3174 CYPRESS
0137 NEWPORT
0145 OCONEE
0007 ANDERSON
0007 ANDERSON
0068 ENO
0165 PLEASANT GARDEN
0068 ENO
0088 HODGES 
MZA Utility Consultants

12228 Venice Blvd. Suite 397

Los Angeles, CA 90066

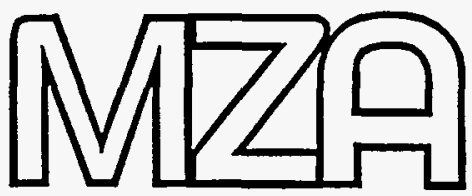

January 15, 1993

University of California

Los Alamos National Laboratory

Attn: J.F. Roach

Reference: 9-LY2-W6097-1

Mail Stop 299

P.O. Box 1663

Los Alamos, NM 87545

Dear Mr. Roach:

Subject: Transmittal of Italian Power Flow Model

Enclosed are three copies of the Italian State (ENEL) AC power flow model and related database. The model was constructed from data received from the Italian National Control Center (a sample map and hand drawn diagram of their mimic board are attached), and the western European reliability council, UCPTE (a two page intertie table is attached). The information was acquired during a 1978 business trip that included a day long visit to the ENEL national control center. The original model was refined during demonstrations performed for control center personnel in 1989 and 1990. The final model reflects data gathered during a short visit to the control center in 1991.

For simplification, $220 \mathrm{kV}$ interties have been modeled into the $380 \mathrm{kV}$ system. The Grande-Ile substation shown on the map is actually connected to the Albertville (F) substation. Laufenburg substation is included to represent power transfers from northern European systems.

The model will diverge using the decoupled (DSOLVE) algorithm. It will diverge after 6 iterations the first time the Newton (NSOLVE) algorithm is used but will converge quickly on the second attempt. I have found this to be an idiosyncrasy of the solution method not the model.

A copy of this letter and all attachments except the model, database and hand drawn diagram have been forwarded to Mr. Michael Pierotti.

If you have any questions, please contact me at (213) 299-7561.

Thank you;

Michele Wynne

Principal 
(31 DECEMBER 1987)

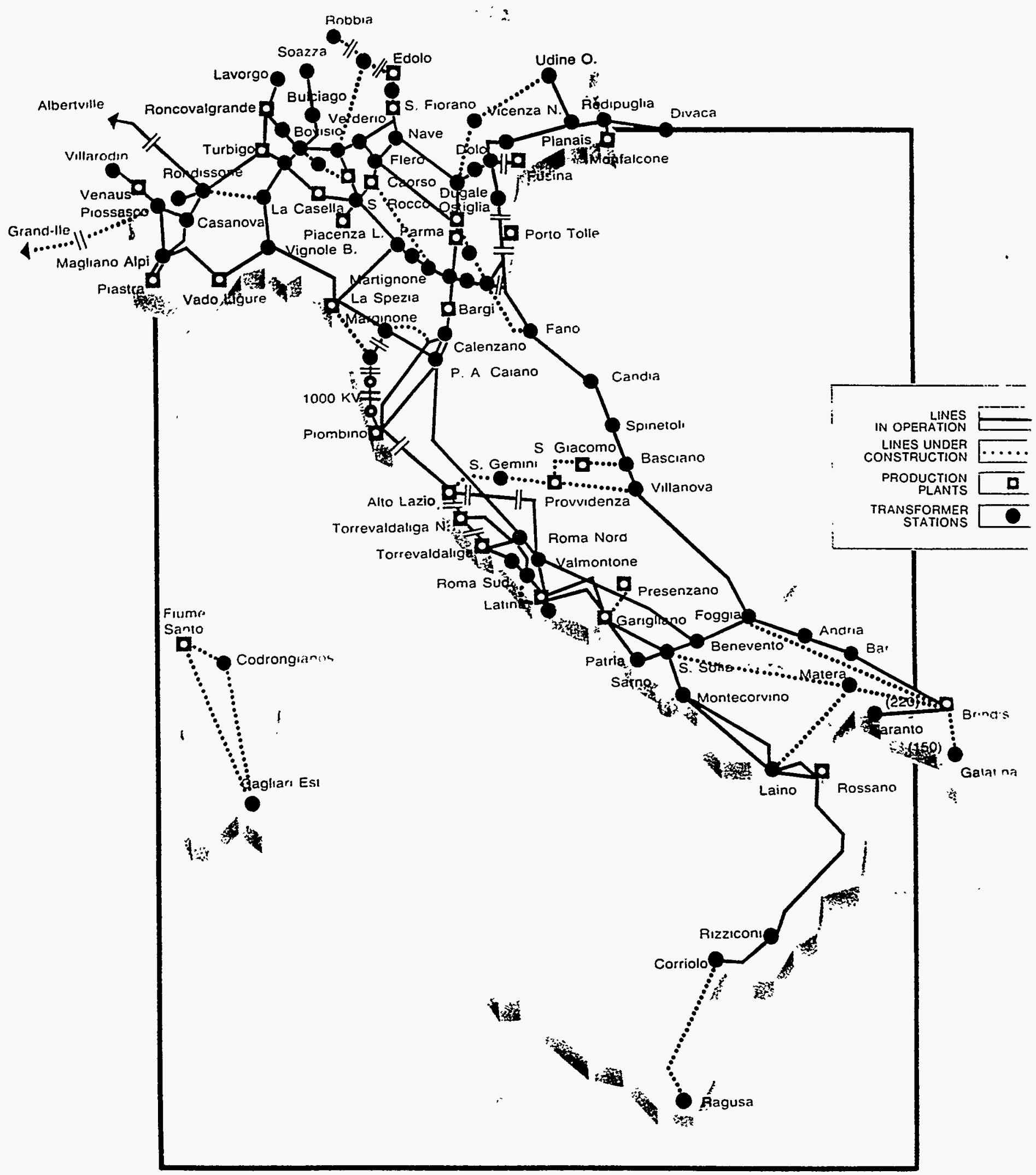




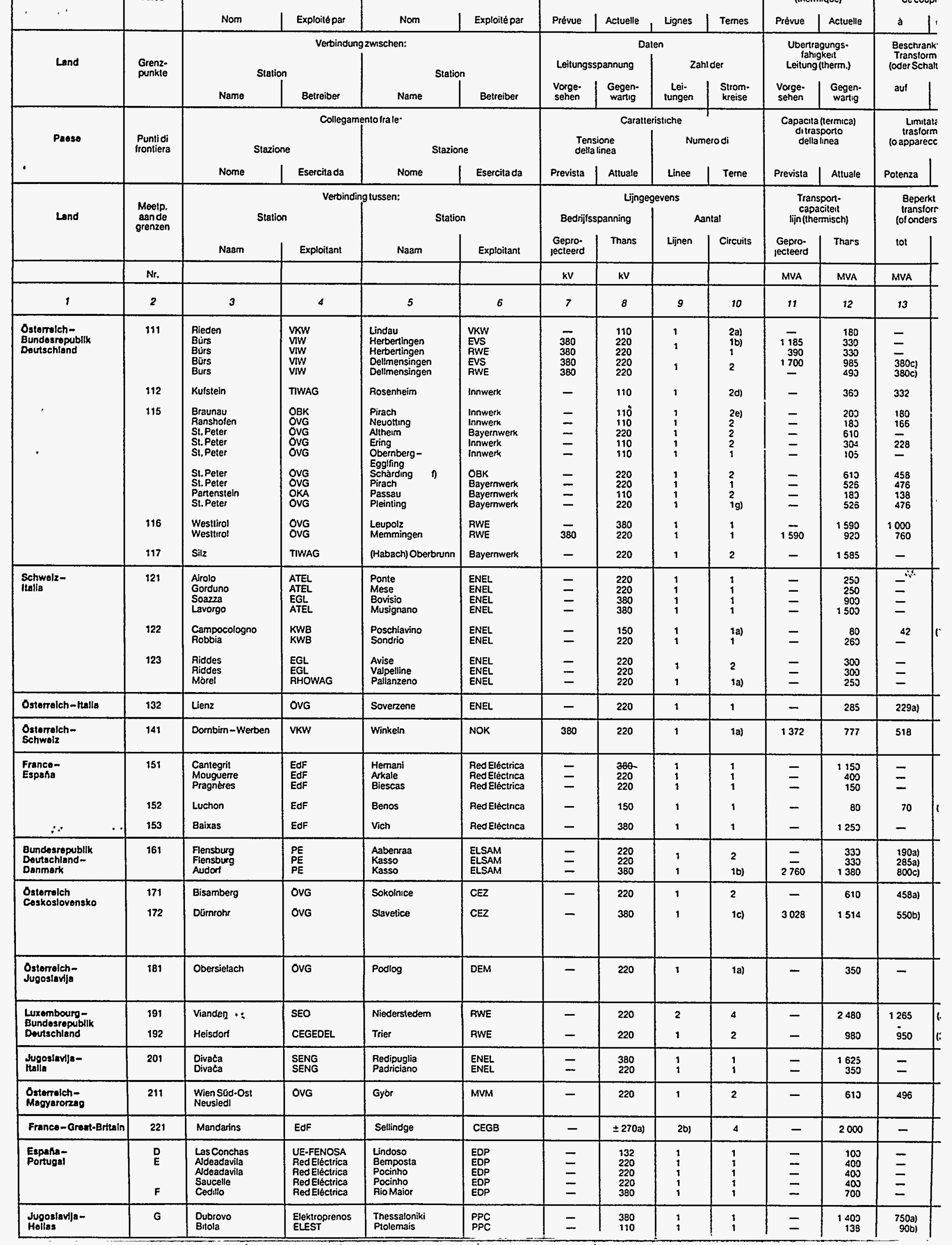


